\title{
Managing insomnia: an overview of insomnia and pharmacologic treatment strategies in use and on the horizon
}

\author{
Thomas L Schwartz, Viral Goradia \\ SUNY Upstate Medical University, Psychiatry Department, Syracuse, NY 13210, USA
}

\begin{abstract}
This review explores basic sleep physiology, the mechanism of action for each class of hypnotic agents, their clinical application based on pharmacodynamic and pharmacokinetic factors, and potential pharmacologic sleep-inducing mechanisms of future hypnotics. The paper challenges the reader to understand the neuroscientific basis of insomnia and use this knowledge to guide prescription of hypnotic agents.

Currently indicated hypnotic agents are discussed with regard to their mechanism of drug action and clinical application. A broader discussion is developed throughout this paper regarding other non-indicated agents that may improve sleep and describing newer pharmacological treatments that may become available in the future for use in sleep disorders and their comorbid conditions.

Keywords: hypnotic agents, insomnia, sleep, sedatives.

Citation

Provenance

Dates

Copyright

Correspondence address

Email address

Competing interests

Abbreviations

Schwartz TL, Goradia V. Managing insomnia: an overview of insomnia and pharmacologic treatment strategies in use and on the horizon. Drugs in Context 2013; 212257. doi: 10.7573/dic.212257

Invited; externally peer reviewed

Submitted: 4 September 2012; Accepted, subject to peer review: 6 September 2012; Revised manuscript submitted: 30 August 2013; Published: 4 October 2013

(c) 2013 Schwartz TL, Goradia V. This is an open-access article distributed under the terms of the Creative Commons Attribution License (CC-BY-NC-ND 3.0) which allows unrestricted sharing, copying and distribution for personal use provided it is properly attributed. No other uses without permission.

Thomas L Schwartz, MD, SUNY Upstate Medical University, Psychiatry Department, 750 East Adams Street, Syracuse, NY 13210, USA schwartt@upstate.edu

The authors have declared the following competing interests: Dr. Schwartz receives grant support from Teva, Cyberonics and Bristol-Myers Squibb. Dr. Goradia has no conflicts to disclose.

5HT2, serotonin; ADHD, attention deficit hyperactivity disorder; BZRA, benzodiazepine receptor agonists; cAMP, cyclic adenosine monophosphate; CB1, cannabinoid receptor type; $C B 2$, cannabinoid receptor type; CNS, central nervous system; $\mathrm{DA}_{2}$, dopamine; DEA, Drug Enforcement Agency; DLPFC, dorsolateral prefrontal cortex; DSM-5, Diagnostic and Statistical Manual 5; GABA, gamma-amino butyric acid; $\mathrm{GABA}_{\mathrm{A}^{\prime}}, \mathrm{GABA}$ receptor type $\mathrm{A} ; \mathrm{GABA}_{\mathrm{B}^{\prime}} \mathrm{GABA}$ receptor type $\mathrm{B} ; \mathrm{GHB}$, $\gamma$-hydroxybutyric acid; $\mathrm{H} 1$, histamine- 1 ; $L C$, locus ceruleus; MT1, melatonin receptor type 1; MT2, melatonin receptor type 2; NK , neurokinin receptor type 1; NREM, non-rapid eye movement; OX1, orexin-1; OX2, orexin-2; PAM, positive allosteric modulation; $\mathrm{PK}_{2}$, prokineticin 2; PTSD, post-traumatic stress disorder; RAS, reticular activating system; REM, rapid eye movement; SCN, suprachiasmatic nucleus; Substance P, NK1; SWA, slow wave activity; SWS, slow wave sleep; TMN, tuberomammillary nucleus; VLPO, ventrolateral preoptic.
\end{abstract}

\section{Introduction}

Insomnia is a common disorder and, in the realm of psychiatric care, one of the most common residual symptoms in major depression after antidepressant treatment is offered [1]. Insomnia is a commonly induced side-effect as well. Insomnia can be an acute predictor of who will ultimately attempt suicide [2]. It continues to be a clinical target symptom of interest for clinicians who treat psychiatric conditions, medical conditions, pain conditions, or associated sleep disorders. There are clearly indicated hypnotic agents and many off-label medications that may be used to improve sleep duration or quality. This offers an opportunity for clinicians to engage in rational polypharmacy

Drugs in Context - The journal of interventions in clinical practice This full text PDF downloaded from www.drugsincontext.com Drugs in Context 2013; 212257. ISSN 1740-4398

Copyright (C) 2013 Schwartz TL, Goradia V. Distributed under the terms

of the Creative Commons Attribution License CC-BY-NC-ND 3.0.

No other uses without permission. in order to treat insomnia alone or when part of a comorbid condition [3]. The most widely used prescription hypnotic agents since the 1960s have utilized the same mechanism of action to induce sleep. Here, gamma-amino butyric acid (GABA) neuronal activity is enhanced by positive allosteric modulation (PAM). Classically, if $\mathrm{GABA}_{\mathrm{A}}$ receptor PAM occurs in wakefulness brain centers, alertness falters and sleep prevails. Alternatively, if $G_{A B A}$ receptor activity increases in sleep-promoting centers, then sleep prevails more directly [4]. The classic benzodiazepine hypnotic agents work through both of these mechanisms, as do the barbiturates and ethanol, and will be further discussed later in this article.

The use of $\mathrm{GABA}_{\mathrm{A}}$-modulating agents has been effective for treating insomnia, but at a cost of an adverse effect profile consisting of addiction, over-sedation, psychomotor impairment, respiratory suppression, and somnambulism. The development of tolerance and the need for progressively higher doses, physiological and psychological dependence on medications, 
and potential withdrawal from medications can cause significant difficulties for patients. $\mathrm{GABA}_{\mathrm{A}}$ receptors are ubiquitous and facilitating them is akin to a blanketing effect where neuronal dampening occurs across the CNS, not necessarily localized to sleep-wake centers. The need for treating insomnia as a primary entity or as a secondary symptom of mental or medical disorder continues, as does the need to find agents that are more specific for sleep-wake neurocircuitry with less risky adverse effects.

\section{Overview of sleep-wake neurocircuitry}

Neurotransmitter systems (histamine, acetylcholine, norepinephrine, serotonin, orexin, and dopamine) may promote alertness, wakefulness, and vigilance when activated and contrarily could become hypnotic and sleep inducing if they were antagonized, thus removing arousal in the CNS. One prototypical circuit is the 'sleep-wake switch'. When one side of the switch is active patients are awake and alert; when the other side is active patients go to sleep. Approved hypnotic agents have relied heavily on manipulating the $\mathrm{GABA}_{\mathrm{A}}$ receptor and its neurocircuitry. These $\mathrm{GABA}_{\mathrm{A}}$ receptorenhancing drugs effectively turn on sleep promoting areas of the brain and inhibit arousal centers. Agents that are largely used 'over-the-counter', or prescribed as off-label treatments, utilize the antagonism of the histamine system. Blocking histamine-1 (H1) receptors successfully dampens cortical arousal, and fatigue and sleepiness result. Diphenhydramine, chlorpheniramine, and doxylamine have all been approved for over-the-counter hypnotic use and are often incorporated into pain, cold, and influenza remedies. Psychopharmacologists often use off-label, sedating antidepressants such as trazodone, mirtazapine, or amitriptyline to induce sleep as they typically utilize $\mathrm{H} 1$ receptor antagonism as well [5]. Recently, the aged tricyclic antidepressant doxepin has been approved as the first antihistamine prescription sleeping agent, being used at low doses to induce sleep [6]. The final existing prescription hypnotic agent, ramelteon utilizes a unique mechanism and facilitates the melatonin system's activity [7]. This drug agonizes the melatonin type 1 (MT1) and melatonin type 2 (MT2) receptors and this activity is thought to dampen reticular activating system monoamines (norepinephrine, serotonin, and dopamine) from arousing the cortex and to correct circadian rhythm activity respectively. The suprachiasmatic nucleus (SCN) is similar to a master switch which helps to control and guide the simple 'sleep-wake switch'. Melatonin is released as the SCN signals darkness is present and then the simple switch is pushed towards promoting sleepiness. Similarly, if ramelteon is taken, melatonin receptors are also directly stimulated and the switch is moved as if endogenous melatonin is present.

The authors fully expect that hypnotic agents will continue to utilize the properties of $\mathrm{GABA}_{\mathrm{A}}$ receptor agonism, melatonin receptor agonism, or histamine receptor antagonism well into the future. This paper serves next to review current hypnotic treatments in more detail and then to focus upon off-label medication findings with regard to improving sleep quantity and quality. Finally, future-oriented treatments for the treatment of insomnia will be discussed.

\section{Diagnostic and Statistical Manual (DSM-5)}

The DSM-5 has set criteria for the diagnosis of insomnia disorder [8], a predominant complaint of dissatisfaction with sleep quantity or quality, associated with one (or more) of the following symptoms:

1. Difficulty initiating sleep. (In children, this may manifest as difficulty initiating sleep without caregiver intervention.)

2. Difficulty maintaining sleep, characterized by frequent awakenings or problems returning to sleep after awakenings. (In children, this may manifest as difficulty returning to sleep without caregiver intervention.)

3. Early-morning awakenings with inability to return to sleep.

4. The sleep disturbance causes clinically significant distress or impairment in social, occupational, educational, academic, behavioral, or other important areas of functioning.

5. The sleep difficulty occurs at least 3 nights per week.

6. The sleep difficulty is present for at least 3 months.

7. The sleep difficulty occurs despite adequate opportunity for sleep.

8. The insomnia is not better explained by and does not occur exclusively during the course of another sleep-wake disorder (e.g., narcolepsy, a breathing-related sleep disorder, a circadian rhythm sleep-wake disorder, a parasomnia).

9. The insomnia is not attributable to the physiological effects of a substance (e.g., a drug of abuse, a medication).

10. Coexisting mental disorders and medical conditions do not adequately explain the predominant complaint of insomnia.

The DSM-5 has removed the diagnosis of primary insomnia, replacing it with insomnia disorder. The diagnosis may be given when comorbid with another mental disorder, sleep disorder, or medical condition. When the insomnia is severe enough to warrant independent clinical attention, the concurrent diagnosis of insomnia disorder should be made.

\section{Contemporary treatment of insomnia}

\section{Medical management}

The following medication classes presented in historical order have been approved for the short-term and sometimes long-term management of insomnia [4,5,9]:

\section{Barbiturates}

Although barbiturates have many potential drawbacks such as addiction, drug interactions, lethality in overdose, and cognitive impairment that prevent them from being commonly prescribed, they may be used for short-term treatment of insomnia on rare occasions. However, safer treatments are clearly available and should be considered before the use of barbiturates. They are discussed here given their historical significance in the armamentarium of available hypnotic agents.

Amobarbital, butabarbital, pentobarbital, secobarbital, and phenobarbital may be considered for their hypnotic effects. Of these, phenobarbital has a long duration of action as compared to the rest, which have an intermediate duration of action. 


\section{Mechanism of action}

With regard to sleep circuitry, barbiturates act by increasing the frequency and duration of opening of the chloride ion channel in the $\mathrm{GABA}_{\mathrm{A}}$ receptor complex through a binding site separate from that of benzodiazepines. The requirements for the facilitation of GABA-based neurotransmission require a GABA molecule and a barbiturate molecule to bind concomitantly onto their specific sites on a $\mathrm{GABA}_{\mathrm{A}}$ receptor. The barbiturate makes this complex much more efficient and more active, essentially increasing the function of the receptor's ligand-gated channel. Effectively, a greater amount of chloride ion influx into the neuron is allowed more quickly compared to a receptor without a barbiturate bound to it. This increased activity and facilitation by addition of a medication is called positive allosteric modulation (PAM). The abrupt excess of activity in the sleep-wake switch can quickly produce sleep by enhancing the sleep-promoting half of the switch and dampening the arousal half simultaneously.

\section{Adverse drug reactions}

Barbiturate effects on the CNS follow a pattern: calming progresses to sleep to unconsciousness to coma to surgical anesthesia and finally, fatal respiratory and cardiovascular depression in a dose-dependent fashion. The therapeutic index for the sedative effects of the barbiturates is low and fatal overdose may occur with consumption of too many pills dispensed in a single prescription. In addition, barbiturates are known to produce both a cross-tolerance to other sedating drugs acting on $\mathrm{GABA}_{\mathrm{A}}$ receptors (including alcohol and the benzodiazepines) and be addictive in nature. In case of respiratory arrest, reversal with flumazenil is ineffective. Studies on the use of barbiturates for short-term or long-term treatment of insomnia are relatively insufficient compared to clinical studies or data available to guide prescribing strategies. There are also significant risks to this class of medications, therefore they are not recommended any more for the treatment of insomnia [10].

\section{Benzodiazepines}

In an analysis of West Virginia Medicaid data from 2003 regarding prescription hypnotic agents (including off-label use of the antidepressant trazodone), the benzodiazepines accounted for almost $11 \%$ of claims filed (estazolam $0.4 \%$, flurazepam $0.1 \%$, temazepam $10 \%$, and triazolam $0.3 \%$ ) [11]. In contrast, zolpidem accounted for $42.7 \%$ of claims, zaleplon $1 \%$, and trazodone $45.5 \%$. The $11 \%$ use of the traditional benzodiazepines accounts for a minority, but reasonable market share with regard to utilization. Furthermore, it is suspected that anxiolytic benzodiazepines that are not approved for insomnia (alprazolam, clonazepam, lorazepam, diazepam) are prescribed off-label for this purpose.

\section{Mechanism of action}

Classical hypnotic benzodiazepines (flurazepam, temazepam, triazolam and quazepam) act as PAMs of the $\mathrm{GABA}_{\mathrm{A}}$ receptors binding to their own sub-site on the $\mathrm{GABA}_{\mathrm{A}}$ receptor similar to the barbiturates. Their net effect is to increase the sensitivity and activity of the $\mathrm{GABA}_{\mathrm{A}}$ receptor/GABA complex that increases the frequency of opening of the central chloride channels. In a sense,
PAM makes the receptor-transmitter-ion channel complex more active with more openings at any given time, thus being more efficient at lowering neuronal activity. If this decrease in neuronal firing occurs in wakefulness centers, then somnolence may occur.

The benzodiazepine chemically structured agents have the ability to promote sleep, dampen anxiety, and provide for muscle relaxation. Their ability to rapidly induce sleep in prototypical phobic insomniacs and ability to lower anxiety quickly, makes these drugs very reinforcing where patients believe they need the agent to sleep, and cannot do so without it. This may lead to overuse, addiction, or dependence in a minority of patients.

\section{Drug selection}

The choice of which benzodiazepine to use is generally made on a case-by-case basis with the rapidity of absorption, onset of action, and half-life of the drug being the most important considerations.

For example, in patients who report difficulty in initiating sleep, a drug with a rapid onset of action is preferred, whereas a drug with a longer half-life may be required in patients who report sleep fragmentation, poor maintenance of sleep, or earlymorning awakening as their major concern.

Flurazepam has a rapid onset of action, achieving peak plasma concentrations in 0.5 to 1 hour. Triazolam has an onset of about 0.5 hours [12]. Quazepam (1.5 hours) has an intermediate onset of action. Temazepam in its original preparation had an onset of action of about 3 hours and therefore would not be preferred in a patient having difficulty in initiating sleep [13]; however, it is available in capsule form that is rapidly absorbed through the GI tract and which has an onset of about 0.5 to 0.75 hours and reaches peak concentration in about 1.5 hours [12].

Drugs with a longer half-life (flurazepam and quazepam) are advantageous in reducing early-morning awakenings on repeated administration. However, this may also lead to unwanted cumulative dosing side-effects, including daytime drowsiness, cognitive impairment, and interactions with other CNS depressants and alcohol. In the elderly, the accumulation of the metabolites of these long-acting drugs may be even greater and, therefore, predispose to the development of intoxication and delirium.

In contrast, short-acting benzodiazepines, such as triazolam, tend not to accumulate but may lead to rebound insomnia upon discontinuation, especially if used consecutively over several weeks.

\section{Adverse drug reactions}

Benzodiazepines may cause tolerance, anterograde amnesia, sleep walking behaviors, delirium, cognitive impairment, fall and fracture risk, and respiratory drive suppression.

Very short-acting benzodiazepines, such as midazolam and triazolam, are known to cause amnesia. In a review of doubleblind, placebo-controlled trials, neuropsychological testing performed on subjects before and after administration found that short-acting benzodiazepines, such as midazolam, caused decreased performance on both amnestic and non-amnestic cognitive tests [14]. Cases of next-day memory impairment and amnesia have been reported with triazolam [15]. Triazolam has been shown to cause deficits in information acquisition and recall, or anterograde amnesia, both 1.5 hours following 
the dose of triazolam, and more markedly 24 hours after the dose in a dose-dependent manner [12]. Cases of auditory and visual hallucinations after administration of triazolam have also been reported [16].

\section{Benzodiazepine receptor agonists (BZRA or 'Z' drugs) \\ Mechanism of action}

These benzodiazepine-like drugs are so called as they tend to begin with the letter ' $Z$ ' (zolpidem, zaleplon, eszopiclone) and produce 'Z's or sleep; they are also PAMs of the GABA $A_{A}$ receptor. They do not share the classical benzodiazepine structure, but they exert similar effects upon the GABA $A_{A}$ receptor. However, they differ in the sense that they demonstrate selective binding to the GABA receptor's alpha-1 subunit at prescribed doses. The alpha-1 subunit has been known to be critical and more selective for producing acute sedation and somnolence. The alpha- 1 subunit is also linked to daytime sedation, anti-convulsant actions, and possibly to amnesia, which may produce these adverse effects that are common in the true benzodiazepines. The net effect is that these drugs have less anxiolytic and muscle relaxant properties (usually associated with alpha-2 and/or alpha-3 subtypes) and are more specific for hypnotic effects [4]. The addiction risk and Drug Enforcement Agency (DEA) addiction labeling is considered less severe than the benzodiazepines.

Zaleplon and zolpidem are very alpha-1 selective. In contrast, eszopiclone is less selective and has more resemblance to true benzodiazepines as it shows a balanced selectivity for multiple different alpha subunits.

\section{Drug selection}

The choice of the drug, once again, is made keeping in mind specific patient requirements and the pharmacokinetics of each drug. Eszopiclone has the longest half-life ( -6 hours) as compared to zolpidem ( $\sim 1-2$ hours) and zaleplon ( $\sim 1$ hour) [13]. Also, zaleplon has the fastest onset of action, followed by zolpidem and eszopiclone. The onset of action is typically accelerated if these drugs are taken on an empty stomach. Zaleplon, therefore, is preferred in patients who have trouble initiating sleep and eszopiclone may be a better choice in patients who have difficulty maintaining sleep. Zaleplon can be taken for middle insomnia as long as 4 hours remain before awakening. More recently, a sublingual zolpidem preparation has become similarly available for middle-of-the-night insomnia symptoms. Eszopiclone is approved for more chronic use. Zolpidem controlled release is also approved for chronic use and its slow release preparation artificially increases its half-life and maintains drug plasma levels further into the night.

\section{Adverse drug reactions}

Both benzodiazepines and the ' $\mathrm{Z}$ ' drugs are known to cause tolerance and have some potential for abuse along with cross-tolerance to alcohol and other sedatives linked to the $\mathrm{GABA}_{\mathrm{A}}$ receptor.

Cases of auditory hallucinations, visual hallucinations, formication, and delusions have been reported with the use of ' $\mathrm{Z}$ ' drugs at recommended doses. Multiple cases of visual hallucinations, auditory hallucinations, and delusions have been reported with the use of zolpidem [17-19]. Two of these cases occurred following the first dose at recommended dosage. A patient on eszopiclone was reported to have developed formication, delusions, and visual hallucinations. He developed hallucinations of bugs on his skin and in his home, had his landlord spray for bugs repeatedly, and his symptoms worsened when the dose was increased and resolved within a week of discontinuing the medication [20]. In March 2007, the FDA required a warning be added to all sedative-hypnotics regarding severe allergic reactions and complex sleep behaviors, such as sleep driving or preparing food while asleep. Two cases were reported of spousal homicide after ingesting higher than recommended dosages of zolpidem [21]. Forensic psychiatrists for the prosecution and defense differed on their views of the role zolpidem played in the killings. The patients had full or partial amnesia of the events and the night of the killings. Numerous cases of sleep driving have been reported while taking BZRAs, as well as cases of sleep walking, sleep eating, sleep conversations, sleep sex, and sleep shopping [22,23]. It is unclear if these drugs have the same fall potential and respiratory suppression potential as the benzodiazepine agents. As doses are escalated above the norm, or if these agents are mixed with alcohol or opiates, these types of side-effect risks likely increase dramatically.

\section{Antihistamine drugs}

Thesedrugsincludeover-the-countersleepaids (diphenhydramine and doxylamine) and prescription doxepin.

\section{Mechanism of action}

As noted, blocking histamine-1 receptors in the cortex and ventrolateral preoptic (VLPO) nucleus in the hypothalamus turns the sleep-wake switch off. The absence of the stimulating effect of histamine in these areas ultimately promotes sedation and sleep.

A prescription antihistamine medication approved for improving sleep maintenance in the treatment of insomnia is the tricyclic antidepressant doxepin, which inhibits serotonin and norepinephrine uptake pre-synaptically and is an antagonist at histamine-1, muscarinic-1, and alpha-1 adrenergic receptors at high doses (150-300 mg). However, doxepin has been found to be quite selective for antagonizing histamine-1 receptors at low doses (3-6 mg), thus allowing for safe use in insomnia [4]. Theoretically, the selective anti-histaminic action at lower doses for mirtazapine, trazodone, and quetiapine as well may provide off-label effectiveness for insomnia [4].

\section{Adverse drug reactions}

These drugs may be associated with anti-cholinergic side-effects (blurred vision, constipation, memory problems and dry mouth). They tend to have longer half-lives and can produce morning sedation and dizziness.

As compared to benzodiazepines, drugs antagonizing the histamine-1 receptor have been found to have significantly lower, to no, risk for addiction, abuse, ataxia and respiratory suppression. 


\section{Melatonin agonists}

Ramelteon is an agonist at MT1 and MT2 receptors. The agonism of melatonin at MT1 and MT2 is thought to dampen the activity of the reticular activating system and its monoamines (serotonin, dopamine and norepinephrine), thereby promoting sleep [4]. It is has been known to be useful in initiating sleep with no clear effect on maintenance of sleep. Ramelteon is known to not cause addiction or withdrawal and has no abuse potential as compared to benzodiazepines or BZRA drugs. It can have limited bioavailability in some patients which can lead to ineffectiveness.

Over-the-counter melatonin has been shown to decrease sleep onset latency as well as improve subjective sleepiness upon awakening, although it did not improve scores of sleepiness, fatigue, and alertness throughout the day in more formal studies [24]. It likely has a high degree of first pass liver metabolism and it is unclear how much penetrates the blood-brain barrier, making bioavailability also questionable.

\section{Valerian}

Although studies on valerian have yielded conflicting results [25], it has been shown to improve sleep quality in post-menopausal women [26]. In general, the evidence shows no benefit compared with placebo when studied more stringently. There have been case reports of patients with hepatotoxicity when taking products containing valerian [10].

\section{Novel hypnotic approaches}

\section{Serotonin systems}

The serotonin (5HT2) receptor subfamily is comprised of several types with the three most commonly studied subtypes: $5 \mathrm{HT}_{2 \mathrm{~A}}$, $5 \mathrm{HT}_{1 \mathrm{~A}}$, and $5 \mathrm{HT}_{2 \mathrm{C}}$. Evidence from both clinical and preclinical studies suggests that $5 \mathrm{HT}_{2 \mathrm{~A}}$ receptors modulate slow wave sleep (SWS), which is deep and restorative in nature and often lessened in the midst of major depressive disorder [27,28]. These agents may not induce hypnosis, or sleep onset, but once sleep occurs, there is a shift towards more efficient and improved SWS. $5 \mathrm{HT}_{2 \mathrm{~A}}$ receptor blockade might promote better sleep by a complex mechanism. Serotonin typically diminishes cortical glutamatergic arousing neurons by agonizing $5 \mathrm{HT}_{1 \mathrm{~A}}$ receptors and enhances glutamatergic excitatory arousal by stimulation of $5 \mathrm{HT}_{2 \mathrm{~A}}$ receptors. In effect, antagonizing the $5 \mathrm{HT}_{2 \mathrm{~A}}$ receptor dampens cortical activity to promote somnolence and fatigue. This may help maintain deeper sleep throughout the night as normal sleep cycle arousal is lowered. Currently there are several compounds (volinanserin, eplivanserin, esmirtazapine, pruvanserin, pimavanserin, APD125, AVE8488, HY-10275, ITI-722) in clinical development for the treatment of insomnia that have $5 \mathrm{HT}_{2 \mathrm{~A}}$ antagonism, at least in part, as their hypnotic mechanism [29-32]. The activation of the $5 \mathrm{HT}_{2 \mathrm{~A}}$ receptor could exert a tonic inhibition of SWS [33]. A study of a potent and selective $5 \mathrm{HT}_{2 \mathrm{~A}}$ receptor antagonist showed that a single oral dose resulted in a massive increases of SWS and slow wave activity (SWA). Antagonism of $5 \mathrm{HT}_{2 \mathrm{~A}}$ receptors enhanced SWA and reduced spindle frequency activity [33]. Improvement in SWS may contribute to the effects that atypical antipsychotics have on sleep, as these medications are $5 \mathrm{HT}_{2 \mathrm{~A}}$ antagonists. Oftentimes, the atypical and the sedating antidepressants (trazodone, mirtazapine) have this common $5 \mathrm{HT}_{2 \mathrm{~A}}$ receptor antagonism plus the common property of histamine $\mathrm{H} 1$ receptor antagonism. This latter effect, as noted from previous sections, does induce sedation and sleepiness and likely, the $5 \mathrm{HT}_{2 \mathrm{~A}}$ receptor antagonism promotes deeper sleep after hypnosis occurs.

\section{Orexin system}

The neuropeptides orexin-A and orexin- $\mathrm{B}$ (also known as hypocretin-1 and -2) act as endogenous ligands and bind to the orexin-1 (OX1) and -2 (OX2) receptors that are found in the lateral hypothalamus, which then project throughout the brain. The orexin system plays an important role in the regulation of sleep and wakefulness, especially in the maintenance of sustained wakefulness. Modafinil and armodafinil are drugs that potentially agonize this system and are indicated for promoting wakefulness in narcolepsy, apnea, and shift work patients [34,35]. Orexin neurons link the ventrolateral preoptic area and the brainstem's reticular activating system's wakefulness-promoting nuclei (serotonin, norepinephrine, and dopamine). This linkage stabilizes behavioral states by activating arousal regions during wakefulness and preventing unwanted fatigue and somnolence intrusions during traditional wakefulness periods of the day. Orexin neurons discharge during active waking and cease firing during sleep. Therefore, the effect of an orexin receptor antagonist could theoretically remove arousal and wakefulness thus inducing sleep and reducing nocturnal wakefulness in patients with insomnia [36,37].

Almorexant is an OX1 and OX2 receptor antagonist. Patients or animals deficient in orexin may develop sleep attacks or narcolepsy. It follows that removing orexin activity by blocking OX1/2 receptors may produce onset of sleep [38]. Preliminary studies had shown that this agent was potentially effective in treating insomnia but development has recently ceased due to safety concerns $[36,39]$. Other antagonists appear to be in development however.

\section{Neurokinin system}

The development of $\mathrm{NK}_{1}$ receptor antagonists started in the 1990s following an intensive decade of research in pharmacology, biotechnology, and medicinal chemistry. The localization and roles of $\mathrm{NK}_{1}$ receptors were oriented mainly towards targets related to pain, depression, anxiety, or emesis [40]. $\mathrm{NK}_{1}$ agonism in rodents facilitates dopamine transmission, which is associated with alertness, wakefulness, vigilance, anxiety, aggression, and rage [36].

In animal models, $\mathrm{NK}_{1}$ receptor antagonism is associated with the opposite effect, anxiolysis [41]. As insomnia is often driven by anxiety and hyperarousal, this model may provide a preclinical rationale for an anxiety or insomnia treatment. $\mathrm{NK}_{1}$ is also known as substance $P$, and research regarding pain and depression have been largely negative. With regard to insomnia, some promise may exist. Substance $\mathrm{P}\left(\mathrm{NK}_{1}\right)$ receptors are widely distributed in the brain and are specifically found in brain regions that regulate emotion (amygdala, hypothalamus, and the periaqueductal gray). They are also found in close association with serotonin and norepinephrine neurons that are targeted by the currently used antidepressant drugs $[42,43]$. Substance P activity is often 
paired with glutamatergic activity and may result in excessive pain, stress response, depression, and anxiety. Hypothetically, insomnia is a state of CNS hyperarousal and lowering $\mathrm{NK}_{1}$ (substance $\mathrm{P}$ ) activity may lower glutamate neuronal excitation, removing clinical arousal and inducing fatigue and likely sleep. $\mathrm{NK}_{1}$ receptors are also found in association with $5 \mathrm{HT}_{2}$ receptors on neurons, and as noted previously, modulation of certain $5 \mathrm{HT}_{2}$ receptors may promote deeper sleep [44].

Casopitant is an inhibitor at $\mathrm{NK}_{1}$ receptors and is likely to induce sleep [45]. The effect of casopitant for insomnia has been investigated in four Phase II clinical trials, enrolling more than 400 patients, and data are pending [46]. Investigations for this product with regard to anti-emetic properties have ceased, but it is unclear whether studies in insomnia are forthcoming or not.

\section{Alpha-2-delta calcium channel subunit systems}

Agents that antagonize neuronal calcium ion channels, such as gabapentin and pregabalin, are currently approved for treating diseases associated with abnormally excessive neuronal firing (epilepsy, neuropathic pain) [47,48]. Gabapentin has been studied and shown to improve SWS, sleep efficiency, and to decrease inappropriate wakefulness during the night. A second study also showed improved psychomotor functioning and improved sleep maintenance [49]. Pregabalin is indicated for treating fibromyalgia. This disease is noted for marked problems with sleep onset and maintenance, and often has alpha wave intrusions which disrupt SWS. Pooled analyses from fibromyalgia studies and generalized anxiety studies in Europe have indicated subjective improvements for insomnia symptoms associated with these disorders [50].

\section{Prokineticin 2 system}

This protein has been identified in significant quantities in the SCN, which was noted previously to promote melatonin release during sleep which removes reticular activating system arousal, allowing fatigue and somnolence at night and promoting 24 hour circadian rhythms which are needed for optimal sleep [51]. Prokineticin $2\left(\mathrm{PK}_{2}\right)$ is controlled by a clock-controlled gene and may function as a critical SCN output molecule responsible for promoting normal circadian rhythms. In animal models, light induces the SCN to promote excess $\mathrm{PK}_{2}$ which activates $\mathrm{g}$ protein systems which often run in parallel and are activated with glutamate receptor signaling, suggesting that $\mathrm{PK}_{2}$ acts either within the circadian apparatus or on its own externally to promote arousal. In theory, agents that antagonize $\mathrm{PK}_{2}$ might remove arousal and promote sleep. Patents are currently filed for agents in this drug class [52].

\section{Norepinephrine system}

Norepinephrine is a wakefulness-promoting neurotransmitter that is synthesized in the locus ceruleus (LC) which projects throughout the brain. As an originating nucleus in the reticular activating system (RAS), norepinephrine may ascend and activate the dorsolateral prefrontal cortex (DLPFC) to promote arousal, awakening, concentration, and vigilance clinically. The LC projects also to the sleep-wake switch's tuberomammillary nucleus (TMN) and VLPO and also to the circadian clock manager, the SCN. Manipulating this noradrenergic system is a common approach in psychopharmacology for the treatment of depression, anxiety, ADHD and off-label for treating nightmares. If insomnia is caused by anxious hyperarousal, repeated facilitation of the noradrenergic system, with a noradrenergic antidepressant for example, may ultimately downregulate and desensitize the noradrenergic system and remove arousal and anxiety. Clinicians can also dampen noradrenergic activity directly to promote improved sleep. Prazosin is an alpha-1 receptor antagonist finding usefulness in reducing PTSD nightmares. Guanfacine and clonidine are used in the treatment of ADHD and utilize alpha-2 receptor agonism, both of which may promote sedation, fatigue and a calming effect which are often seen as side-effects [53]. These agents are often used to treat sleep difficulties that stem from psychiatric illness or psychiatric medication sideeffects in an off-label manner.

\section{Cannabinoid system}

Two types of cannabinoid receptors, $\mathrm{CB}_{1}$ and $\mathrm{CB}_{2}$, have been studied. $\mathrm{CB}_{1}$ receptors are expressed mainly in the central and peripheral nervous system with $\mathrm{CB}_{2}$ in the periphery only. Activation of either receptor leads to an inhibition of adenylate cyclase and a decreased production of cyclic adenosine monophosphate (cAMP). Additionally, $\mathrm{CB}_{1}$ receptors are thought to be negatively coupled to $\mathrm{N}$ - and $\mathrm{Q} / \mathrm{P}$-type voltage-dependent calcium channels and positively coupled to A-type inwardly rectifying potassium channels, which leads, ultimately, to the closing of calcium channels and decreased neurotransmitter release. The net result is dampening of neuronal firing. Cannabinergic synapses display what is called retrograde transmission, where the presynaptic neuron releases neurotransmitters following depolarization. Next, binding of the neurotransmitters at the postsynaptic membrane stimulates synthesis and release of endocannabinoids, such as anandamide. Once in the synapse, they act back towards the presynaptic neuron to signal the cessation of further neurotransmitter release. Generally speaking, cannabinoid agonists are largely inhibiting with regard to serotonin, norepinephrine, and glutamate. As noted before, these systems, when active, tend to promote wakefulness and arousal, and therefore, when inhibited, sleep may occur [54]. Nabilone is a synthetic cannabinoid approved to treat nausea and vomiting in cancer patients and has anecdotal evidence and a small study conducted in fibromyalgia patients suggesting it may promote better sleep [55]. This trial was randomized, but not placebo controlled. It suggested that both nabilone and the comparator, amitriptyline, helped control insomnia, and on certain clinical measures, nabilone was superior. It is unclear if nabilone promoted better sleep directly or through relief of pain associated with fibromyalgia.

\section{Combining neurotransmitter systems \\ Quetiapine}

Some currently available agents are often used as off-label approaches to treat insomnia when it is associated with mental disorder. Quetiapine makes patients somnolent and has been touted as a clinical hypnotic and berated for being costly, with risks of extrapyramidal and metabolic side-effects. It stands out 
as one of the atypical antipsychotics with a high adverse effect rate of somnolence and sedation. In one instance it may treat insomnia, or it may cause sedating side-effects causing the patient to cease its use. This is the clinical dilemma of most sleepinducing agents (approved, prescription, over-the-counter, offlabel or otherwise) that in order to promote sleep, daytime fatigue and somnolence are resultant side-effects. Given these issues and risks quetiapine, however, is an excellent example of an agent that combines neurotransmitter receptor manipulations to affect sleep onset and maintenance. It is an atypical antipsychotic and is approved to treat schizophrenia, bipolar mania, and depression. This agent is frequently prescribed at low doses that are not capable of treating these approved disease states. An analysis of Medicaid data found that up to $86 \%$ of quetiapine doses were sub-therapeutic, likely indicating off-label use for insomnia symptoms [56]. Another study concluded that $50.3 \%$ of all new prescriptions of quetiapine were for sleep [57].

It is theorized that quetiapine is used for, and promotes, sleep initiation through its antihistaminergic properties and antagonistic activity on noradrenergic $\alpha 1$ and $\alpha 2$ receptors, serotonin, and dopamine receptors, with other more hypothesized mechanisms involving $\mathrm{GABA}_{\mathrm{A}}$ receptors. As it is an atypical antipsychotic, its $5 \mathrm{HT}_{2 \mathrm{~A}}$ receptor antagonism promotes normalization of sleep architecture with improvement of REM and NREM deep sleep ratios once sleep occurs. $\alpha 1$ noradrenergic receptor blockade may dampen patient experience of nightmares similar to prazosin findings, and $\alpha 2$ receptor agonism may dampen noradrenergic arousal allowing better sleep. This agent's metabolite, norquetiapine, also has the property of being a potent norepinephrine reuptake inhibitor. This initial noradrenergic excitation may ultimately downregulate and desensitize the norepinephrine arousal pathways, thus theoretically treating anxiety-induced insomnia. Finally, and particularly at higher doses, the antagonism of $\mathrm{DA}_{2}$ (dopamine) receptors has been known to calm agitated and psychotic patients, and may also calm those who are hyperaroused clinically at the time of sleep onset [58].

\section{Trazodone}

Another drug similarly and remarkably used off-label in psychiatry is trazodone. It has very similar pharmacodynamic properties minus the $\mathrm{DA}_{2}$ receptor antagonism afforded to the atypical antipsychotics [59-61]. The hypnotic effects of trazodone in the $25-150-\mathrm{mg}$ dose range are theoretically explained by $\mathrm{H}_{1}$ antihistamine effects, $5 \mathrm{HT}_{2 \mathrm{~A}}$ receptor antagonism, and $\alpha 1$ adrenergic antagonism [59]. Although the use of trazodone to treat insomnia is off-label, it is clinically effective, has a relatively short half-life, and does not cause dependence [59]. In the last decade, trazodone has been shown to be effective in treating primary insomnia and in insomnia associated with depression, anxiety, PTSD, and dementia [62].

\section{$\gamma$-Hydroxybutyric acid (GHB)/ sodium oxybate}

GHB is a naturally occurring GABA metabolite that has been proposed as a neurotransmitter/neuromodulator that acts via its own receptor [63]. Sodium oxybate is the sodium salt of GHB and its exogenous administration results in potential effects that are mostly mediated by $\mathrm{GABA}_{\mathrm{B}}$ receptors at concentrations high enough to elicit binding, which does not occur at endogenous concentrations of GHB [64]. Higher doses can result in an increase in sleep stages 3 and 4 , seizures, coma, memory impairment, and dependence [63]. GHB can be used for the treatment of cataplexy/narcolepsy and has a limited use in alcohol withdrawal and as an anesthetic [63]. In patients with narcolepsy, sodium oxybate increases slow-wave sleep duration and delta power and reduces the number of night-time awakenings with the most common side-effects consisting of headache, dizziness, nausea, and somnolence [64]. Although GHB is interesting from a mechanistic standpoint, its abuse potential as a recreational drug, use as a "date rape" drug, resulting memory impairment, and dangerousness in overdose make it unattractive as a potential widespread treatment for insomnia at this time, as safer alternatives are readily available.

\section{Conclusions}

In summary, this paper describes the pathways or mechanisms promoting normal sleep and wakefulness and discusses agents typically used to promote sleep and treat insomnia. A secondary discussion of usual drug mechanisms of action was utilized to show how each agent might impact the normal sleep-wake circuitry to either better promote or maintain sleep. The authors discussed often used off-label hypnotic treatment options and discussed potential future insomnia treatment options. Agents that facilitate $\mathrm{GABA}_{\mathrm{A}}$ or melatonergic receptor activity will remove arousal or directly promote sleep, whereas agents that dampen glutamate, serotonin, norepinephrine, orexin, or histamine will effectively remove arousal and secondarily allow improved sleep. It is important to note throughout this paper that some agents will improve sleep onset, treating initial insomnia better, while others may improve deep, restorative sleep, allowing for improvement in sleep maintenance. Ideally this should lead to more refreshing sleep and improved functioning the next day. Some agents possess multiple mechanisms of action which may promote both clinical effects and also explain their significant side-effects. The astute prescriber should be aware of regulatory approvals and the available hypnotic agents within these drug classes, but also appreciate the far greater armamentarium for treating sleep difficulties that comes with understanding how certain off-label or experimental medications affect sleep by manipulating neurocircuitry and neuropharmacology.

\section{Acknowledgments}

Dr. Schwartz would like to thank Dr. Umar Siddiqui for his help in logistical planning and compiling of information related to this manuscript Managing insomnia: an overview of insomnia and pharmacologic treatment strategies in use and on the horizon.

\section{References}

1. Nierenberg AA, Husain MM, Trivedi MH, et al. Residual symptoms after remission of major depressive disorder with citalopram and risk of relapse: a STAR*D report. Psychol Med 2010;40:41-50. http://dx.doi.org/10.1017/ $\underline{\text { S0033291709006011 }}$ 
2. Chellappa SL, Araujo JF. Sleep disorders and suicidal ideation in patients with depressive disorder. Psychiatry Res 2007;153:131-6. http://dx.doi.org/10.1016/j.psychres. 2006.05.007

3. Stahl SM. The 7 habits of highly effective psychopharmacologists: overview. J Clin Psychiatry 2000;61:242-3. http:// dx.doi.org/10.4088/JCP.v61n0401

4. Stahl SM. Stahl's essential psychopharmacology: neuroscientific basis and practical applications. Cambridge, UK; New York: Cambridge University Press; 2008.

5. Stahl SM. Essential Psychopharmacology: The Prescriber's Guide. Cambridge, UK: Cambridge University Press; 2005.

6. Scharf M, Rogowski R, Hull S, et al. Efficacy and safety of doxepin $1 \mathrm{mg}, 3 \mathrm{mg}$, and $6 \mathrm{mg}$ in elderly patients with primary insomnia: a randomized, double-blind, placebo-controlled crossover study. J Clin Psychiatry 2008;69:1557-64. http://dx.doi.org/10.4088/JCP.v69n1005

7. Srinivasan V, Pandi-Perumal SR, Trahkt I, et al. Melatonin and melatonergic drugs on sleep: possible mechanisms of action. Int J Neurosci 2009;119:821-46. http://dx.doi. org/10.1080/00207450802328607

8. American Psychiatric Association. Diagnostic and Statistical Manual of Mental Disorders: DSM-5 (5th ed.). Washington, DC: American Psychiatric Publishing; 2013.

9. Smith MT, Neubauer DN. Cognitive behavior therapy for chronic insomnia. Clin Cornerstone 2003;5:28-40. http:// dx.doi.org/10.1016/S1098-3597(03)90033-0

10. NIH State-of-the-Science Conference Statement on manifestations and management of chronic insomnia in adults. NIH Consens State Sci Statements 2005;22:1-30.

11. Roy AN, Smith M. Prevalence and cost of insomnia in a state Medicaid fee-for-service population based on diagnostic codes and prescription utilization. Sleep Med 2010;11:462-9. http://dx.doi.org/10.1016/j.sleep.2009.09.012

12. Greenblatt DJ. Pharmacology of benzodiazepine hypnotics. J Clin Psychiatry 1992;53(Suppl):7-13.

13. Griffiths RR, Johnson MW. Relative abuse liability of hypnotic drugs: a conceptual framework and algorithm for differentiating among compounds. J Clin Psychiatry 2005;66(Suppl 9):31-41.

14. Tannenbaum C, Paquette A, Hilmer S, Holroyd-Leduc J, Carnahan R. A systematic review of amnestic and non-amnestic mild cognitive impairment induced by anticholinergic, antihistamine, GABAergic and opioid drugs. Drugs Aging 2012;29:639-58. http://dx.doi.org/10.1007/BF03262280

15. Bixler EO, Kales A, Manfredi RL, Vgontzas AN, Tyson KL, Kales JD. Next-day memory impairment with triazolam use. Lancet 1991;337(8745):827-31. http://dx.doi. org/10.1016/0140-6736(91)92524-6

16. Goodchild JH, Donaldson M. Hallucinations and delirium in the dental office following triazolam administration. Anesth Prog 2005;52:17-20. http://dx.doi.org/10.2344/00033006(2005)52[17:HADITD]2.0.CO;2

17. Annseau M, Pitchot W, Hansenne M, Gonzalez Moreno A. Psychotic reactions to zolpidem. Lancet 1992;339(8769):809. http://dx.doi.org/10.1016/0140-6736(92)91935-2
18. Van Puijenbroek EP, Egberts AC, Krom HJ. Visual hallucinations and amnesia associated with the use of zolpidem. Int J Clin Pharmacol Ther 1996;34:318.

19. Markowitz JS, Brewerton TD. Zolpidem-induced psychosis. Ann Clin Psychiatry 1996;8:89-91. http://dx.doi. org/10.3109/10401239609148806

20. Selvaraj V, Skaf M, Sharma A, Faroog U, Frederick P. Eszopiclone-induced psychosis. Innov Clin Neurosci 2012;9:11.

21. Paradis CM, Siegel LA, Kleinman SB. Two cases of zolpidem-associated homicide. Prim Care Companion CNS Disord 2012;14(4): PCC.12br01363. doi: 10.4088/ PCC.12br01363. Epub 2012 Aug 23. http://dx.doi. org/10.4088/PCC. $12 \mathrm{br} 01363$

22. Poceta JS. Zolpidem ingestion, automatisms, and sleep driving: a clinical and legal case series. J Clin Sleep Med 2011;7:632-8. http://dx.doi.org/10.5664/jcsm.1468

23. Dolder CR, Nelson MH. Hypnosedative-induced complex behaviors: incidence, mechanisms and management. CNS Drugs 2008;22:1021-36. http://dx.doi. org/10.2165/0023210-200822120-00005

24. Kayumov L, Brown G, Jindal R, Buttoo K, Shapiro CM. A randomized, double-blind, placebo-controlled crossover study of the effect of exogenous melatonin on delayed sleep phase syndrome. Psychosom Med 2001;63:40-8.

25. Salter S, Brownie S. Treating primary insomnia - the efficacy of valerian and hops. Aust Fam Physician 2010;39: 433-7.

26. Taavoni S, Ekbatani N, Kashaniyan M, Haghani H. Effect of valerian on sleep quality in postmenopausal women: a randomized placebo-controlled clinical trial. Menopause 2011;18:951-5. http://dx.doi.org/10.1097/ gme.0b013e31820e9acf

27. Sharpley AL, Solomon RA, Fernando AI, da Roza Davis JM, Cowen PJ. Dose-related effects of selective 5-HT2 receptor antagonists on slow wave sleep in humans. Psychopharmacology (Berl) 1990;101:568-9. http://dx.doi.org/10.1007/ BF02244239

28. Dugovic C, Wauquier A. 5-HT2 receptors could be primarily involved in the regulation of slow-wave sleep in the rat. Eur J Pharmacol 1987;137:145-6. http://dx.doi. org/10.1016/0014-2999(87)90196-8

29. Teegarden BR, Al Shamma H, Xiong Y. 5-HT(2A) inverse-agonists for the treatment of insomnia. Curr Top Med Chem 2008;8:969-76. http://dx.doi. org/10.2174/156802608784936700

30. 5-HT2A Inverse-Agonists for the Treatment of Insomnia. Available at http://www.intracellulartherapies.com/investor/2009_3_10.htm [Last accessed September 6, 2013].

31. Abbas A, Roth BL. Pimavanserin tartrate: a 5-HT2A inverse agonist with potential for treating various neuropsychiatric disorders. Expert Opin Pharmacother 2008;9:3251-9. http://dx.doi.org/10.1517/14656560802532707

32. Eplivanserin soothes insomnia without next morning effects. Available at: www.clinicalpsychiatrynews.com/ search/search-single-view/eplivanserin-soothes-insomnia-without-next-morning-effects/3bcddb6efo.html [Last accessed September 11, 2013]. 
33. Landolt HP, Meier V, Burgess HJ, et al. Serotonin-2 receptors and human sleep: effect of a selective antagonist on EEG power spectra. Neuropsychopharmacology 1999;21:455-66. http://dx.doi.org/10.1016/S0893133X(99)00052-4

34. Golicki D, Bala MM, Niewada M, Wierzbicka A. Modafinil for narcolepsy: systematic review and meta-analysis. Med Sci Monit 2010;16:RA177-86.

35. Bogan RK. Armodafinil in the treatment of excessive sleepiness. Expert Opin Pharmacother 2010;11:993-1002. http:// dx.doi.org/10.1517/14656561003705738

36. Hoever P, de Haas S, Winkler J, et al. Orexin receptor antagonism, a new sleep-promoting paradigm: an ascending single-dose study with almorexant. Clin Pharmacol Ther 2010;87:593-600. http://dx.doi.org/10.1038/clpt.2010.19

37. Minzenberg MJ, Carter CS. Modafinil: a review of neurochemical actions and effects on cognition. Neuropsychopharmacology 2008;33:1477-1502. http://dx.doi. org/10.1038/sj.npp.1301534

38. Brisbare-Roch C, Dingemanse J, Koberstein R, et al. Promotion of sleep by targeting the orexin system in rats, dogs and humans. Nat Med 2007;13:150-5. http://dx.doi. org/10.1038/nm1544

39. Neubauer DN. Almorexant, a dual orexin receptor antagonist for the treatment of insomnia. Curr Opin Investig Drugs 2010;11:101-10.

40. Quartara L, Altamura M, Evangelista S, Maggi CA. Tachykinin receptor antagonists in clinical trials. Expert Opin Investig Drugs 2009;18:1843-64. http://dx.doi. org/10.1517/13543780903379530

41. File SE. NKP608, an NK1 receptor antagonist, has an anxiolytic action in the social interaction test in rats. Psychopharmacology (Berl) 2000;152:105-9. http://dx/doi. org/10.1007/s002130000513

42. Yip J, Chahl LA. Localization of NK1 and NK3 receptors in guinea-pig brain. Regul Pept 2001;98:55-62. http://dx.doi. org/10.1016/S0167-0115(00)00228-7

43. Gobbi G, Cassano T, Radja F, et al. Neurokinin 1 receptor antagonism requires norepinephrine to increase serotonin function. Eur Neuropsychopharmacol 2007;17:328-38. http://dx.doi.org/10.1016/j.euroneuro.2006.07.004

44. Hokfelt T, Pernow B, Wahren J. Substance P: a pioneer amongst neuropeptides. J Intern Med 2001;249:27-40. http://dx.doi.org/10.1046/j.0954-6820.2000.00773.x

45. Available at www.gsk-clinicalstudyregister.com/protocol_ compounds.jsp [Last accessed August 6, 2010].

46. Various studies on GW679769 (Nct00650871, Nct00280423, Nct00280436 and Nct00354809). Available at: www.clinicaltrials.gov Studies NCT00650871, NCT00280423, NCT00280436, NCT00354809) [Last accessed August 6, 2010].

47. Yurcheshen ME, Guttuso T Jr, McDermott M, Holloway RG, Perlis M. Effects of gabapentin on sleep in menopausal women with hot flashes as measured by a Pittsburgh Sleep Quality Index factor scoring model. J Womens Health (Larchmt) 2009;18:1355-60. http://dx.doi.org/10.1089/ jwh.2008.1257
48. Hindmarch I, Dawson J, Stanley N. A double-blind study in healthy volunteers to assess the effects on sleep of pregabalin compared with alprazolam and placebo. Sleep 2005;28: 187-93.

49. Lo HS, Yang CM, Lo HG, Lee CY, Ting H, Tzang BS. Treatment effects of gabapentin for primary insomnia. Clin Neuropharmacol 2010;33:84-90. http://dx.doi.org/10.1097/ WNF.0b013e3181cda242

50. Russell IJ, Crofford LJ, Leon T, et al. The effects of pregabalin on sleep disturbance symptoms among individuals with fibromyalgia syndrome. Sleep Med 2009;10:604-10. http:// dx.doi.org/10.1016/j.sleep.2009.01.009

51. Cottrell GT, Zhou QY, Ferguson AV. Prokineticin 2 modulates the excitability of subfornical organ neurons. J Neurosci 2004;24:2375-9. http://dx.doi.org/10.1523/JNEUROSCI.5187-03.2004

52. Cheng MY, Bittman EL, Hattar S, Zhou QY. Regulation of prokineticin 2 expression by light and the circadian clock. BMC Neurosci 2005;6:17. http://dx.doi.org/10.1186/14712202-6-17

53. Taylor FB, Martin P, Thompson C, et al. Prazosin effects on objective sleep measures and clinical symptoms in civilian trauma posttraumatic stress disorder: a placebo-controlled study. Biol Psychiatry 2008;63:629-32. http://dx.doi. org/10.1016/j.biopsych.2007.07.001

54. Turcotte D, Le Dorze JA, Esfahani F, Frost E, Gomori A, Namaka M. Examining the roles of cannabinoids in pain and other therapeutic indications: a review. Expert Opin Pharmacother 2010;11:17-31. http://dx.doi. org/10.1517/14656560903413534

55. Ware MA, Fitzcharles MA, Joseph L, Shir Y. The effects of nabilone on sleep in fibromyalgia: results of a randomized controlled trial. Anesth Analg 2010;110:604-10. http:// dx.doi.org/10.1213/ANE.0b013e3181c76f70

56. Hartung DM, Wisdom JP, Pollack DA, et al. Patterns of atypical antipsychotic subtherapeutic dosing among Oregon Medicaid patients. J Clin Psychiatry 2008;69:1540-7. http://dx.doi.org/10.4088/JCP.v69n1003

57. Hermes ED, Sernyak M, Rosenheck R. Use of second-generation antipsychotic agents for sleep and sedation: a provider survey. Sleep 2013;36:597-600. http://dx.doi.org/10.5665/ sleep. 2554

58. Wine JN, Sanda C, Caballero J. Effects of quetiapine on sleep in nonpsychiatric and psychiatric conditions. Ann Pharmacother 2009;43:707-13. http://dx.doi.org/10.1345/aph.1L320

59. Stahl SM. Mechanism of action of trazodone: a multifunctional drug. CNS Spectr 2009;14:536-46.

60. Nierenberg AA, Adler LA, Peselow E, Zornberg G, Rosenthal M. Trazodone for antidepressant-associated insomnia. Am J Psychiatry 1994;151:1069-72.

61. Walsh JK, Erman M, Erwin CW, et al. Subjective hypnotic efficacy of trazodone and zolpidem in DSMIII-R primary insomnia. Hum Psychopharmacol 1998;13:191-8. http://dx.doi.org/10.1002/(SICI)10991077(199804)13:3<191::AID-HUP972>3.0.CO;2-X

62. Bossini L, Casolaro I, Koukouna D, Cecchini F, Fagiolini A. Off-label uses of trazodone: a review. Expert Opin Pharma- 
cother 2012;13:1707-17. http://dx.doi.org/10.1517/14656 566.2012 .699523

63. Crunelli V, Emri Z, Leresche N. Unravelling the brain targets of gamma-hydroxybutyric acid. Curr Opin Pharmacol 2006;6:44-52. http://dx.doi.org/10.1016/j. coph.2005.10.001

64. Pardi D, Black J. gamma-Hydroxybutyrate/sodium oxybate: neurobiology, and impact on sleep and wakefulness. CNS Drugs 2006;20:993-1018. http://dx.doi. org/10.2165/00023210-200620120-00004 